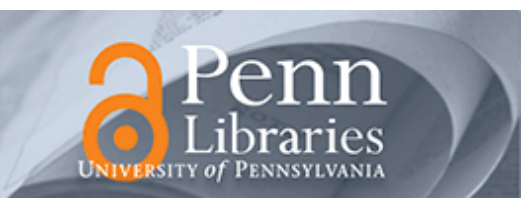

University of Pennsylvania

ScholarlyCommons

2-18-2009

\title{
Reflectionless Sharp Bends and Corners in Waveguides Using Epsilon-Near-Zero Effects
}

\author{
Brian Edwards \\ University of Pennsylvania, brianedw@seas.upenn.edu \\ Andrea Alù \\ University of Pennsylvania; University of Texas at Austin
}

M. G. Silveirinha

Universidade de Coimbra

Nader Engheta

University of Pennsylvania, engheta@ee.upenn.edu

Follow this and additional works at: https://repository.upenn.edu/ese_papers

Part of the Electrical and Computer Engineering Commons

\section{Recommended Citation}

Brian Edwards, Andrea Alù, M. G. Silveirinha, and Nader Engheta, "Reflectionless Sharp Bends and Corners in Waveguides Using Epsilon-Near-Zero Effects", . February 2009.

Suggested Citation:

Edwards, B., Alù, A., Silveirinha, M.G. and Engheta, N. (2009). "Reflectionless sharp bends and corners in waveguides using epsilon-near-zero effects." Journal of Applied Physics. 105, 044905.

(C) 2009 American Institute of Physics. This article may be downloaded for personal use only. Any other use requires prior permission of the author and the American Institute of Physics. The following article appeared in Journal of Applied Physics and may be found at http://dx.doi.org/10.1063/1.3074506

This paper is posted at ScholarlyCommons. https://repository.upenn.edu/ese_papers/583

For more information, please contact repository@pobox.upenn.edu. 


\title{
Reflectionless Sharp Bends and Corners in Waveguides Using Epsilon-Near-Zero Effects
}

\begin{abstract}
Following our recent theoretical and experimental results that show how zero-permittivity metamaterials may provide anomalous tunneling and energy squeezing through ultranarrow waveguide channels, here we report an experimental investigation of the bending features relative to this counterintuitive resonant effect. We generate the required effectively zero permittivity using a waveguide operating at the cutoff of its dominant mode, and we show how sharp and narrow bends may be inserted within the propagation channel without causing any sensible reflection or loss and without affecting its tunneling properties.

\section{Disciplines}

Electrical and Computer Engineering | Engineering

\section{Comments}

Suggested Citation:

Edwards, B., Alù, A., Silveirinha, M.G. and Engheta, N. (2009). "Reflectionless sharp bends and corners in waveguides using epsilon-near-zero effects." Journal of Applied Physics. 105, 044905.

(C) 2009 American Institute of Physics. This article may be downloaded for personal use only. Any other use requires prior permission of the author and the American Institute of Physics. The following article appeared in Journal of Applied Physics and may be found at http://dx.doi.org/10.1063/1.3074506
\end{abstract}




\title{
Reflectionless sharp bends and corners in waveguides using epsilon-near-zero effects
}

\author{
B. Edwards, ${ }^{1}$ A. Alù, ${ }^{1,3}$ M. G. Silveirinha, ${ }^{2}$ and N. Engheta ${ }^{1, a)}$ \\ ${ }^{1}$ Department of Electrical and Systems Engineering, University of Pennsylvania, Philadelphia, \\ Pennsylvania 19104, USA \\ ${ }^{2}$ Department of Electrical Engineering, Instituto de Telecomunicações, Universidade de Coimbra, 3030 \\ Coimbra, Portugal \\ ${ }^{3}$ Department of Electrical and Computer Engineering, University of Texas at Austin, 1 University Station, \\ ENS 616, Austin, Texas 78712-0240, USA
}

(Received 25 July 2008; accepted 19 December 2008; published online 18 February 2009)

Following our recent theoretical and experimental results that show how zero-permittivity metamaterials may provide anomalous tunneling and energy squeezing through ultranarrow waveguide channels, here we report an experimental investigation of the bending features relative to this counterintuitive resonant effect. We generate the required effectively zero permittivity using a waveguide operating at the cutoff of its dominant mode, and we show how sharp and narrow bends may be inserted within the propagation channel without causing any sensible reflection or loss and without affecting its tunneling properties. () 2009 American Institute of Physics.

[DOI: $10.1063 / 1.3074506$ ]

\section{INTRODUCTION}

Metamaterials with near-zero permittivity (epsilon near zero or ENZ) have received increased attention in the last few years for several anomalous properties that characterize their wave interaction. ${ }^{1-9}$ These effects may play an important role in transparency and cloaking phenomena, pattern reshaping, antennas and nanoantennas, nanocircuits, energy squeezing, and supercoupling.

Typically, metamaterials and artificial media may be well described by a "homogenized" effective permittivity and permeability following the same principles that allow any material, inherently a complicated arrangement of atoms and molecules, to be compactly described by its "bulk" properties. However, within metamaterials there is often a second level of homogenization consisting of an artificial intermediate structure whose characteristic size is much greater than the scale of the atoms, but much less than that of the wavelength. The elements of this ordered structure are usually compact resonators that strongly interact with the electromagnetic field, providing anomalous values of effective permittivity and permeability that may not be naturally available. As an alternative realization, one may make use of the dispersive properties of electromagnetic waveguides, substituting the wave interaction of small-scale resonators with the dispersion relations produced by the waveguide walls. ${ }^{9-14}$ For instance, the effective permittivity "experienced" by the dominant transverse-electric $\left(\mathrm{TE}_{10}\right)$ mode in a rectangular metallic waveguide is

$$
\varepsilon_{\mathrm{eff}} / \varepsilon_{0}=n^{2}-c^{2} /\left(4 f^{2} w^{2}\right),
$$

where $w$ is the waveguide H-plane width, $n$ is the relative refractive index of the uniform dielectric filling the waveguide, $c$ is the speed of light in vacuum, and $f$ is the operat-

\footnotetext{
${ }^{a)}$ Electronic mail: engheta@ee.upenn.edu.
}

ing frequency. ${ }^{10}$ For decades, arrays of such waveguides have been employed to realize artificial materials and lenses ${ }^{10}$ with low or negative effective permittivity, and recently the inherently negative effective permittivity provided by a single waveguide below cutoff has been exploited in a variety of applications involving metamaterials. ${ }^{9-13}$ In Ref. 9 in particular, we have applied the effective zero permittivity arising at the waveguide cutoff (at frequency $f=c / 2 w n$ ) to verify experimentally the supercoupling effect and energy squeezing in ultranarrow ENZ channels, as theoretically predicted in our previous works. ${ }^{6,7}$

Indeed, as was theoretically predicted ${ }^{6-8}$ and experimentally verified, 9,10 due to the "static-like" character of the fields in ENZ materials, it may be possible to squeeze and tunnel a significant amount of electromagnetic energy through a very narrow ENZ-filled channel. When such a channel connects two much larger waveguide sections, an anomalous resonance may enable the supercoupling phenomenon, ensuring ultralow phase variation, independent of the length of the ENZ channel and of its specific crosssectional geometry (as long as the longitudinal cross sectional area of the transition region is kept sufficiently small). This phenomenon is drastically different from higherfrequency resonant Fabry-Perot tunneling ${ }^{8}$ and, due to its staticlike features, it is much more robust to geometry variations in the channel. This means that the ultranarrow channel may make arbitrarily sharp turns and be of arbitrary length without affecting its transmission properties.

For a straight channel, we have extensively proven these concepts experimentally using a U-shaped microwave waveguide, in which an ultranarrow channel operated around its cutoff frequency ${ }^{10}$ (and thus behaving effectively as an ENZ material) was connecting two thicker waveguides operated well above their cutoff. Similar experimental proofs have been obtained in a sharp $180^{\circ}$ microstrip bend using resonant complementary split-ring resonator particles to synthesize 


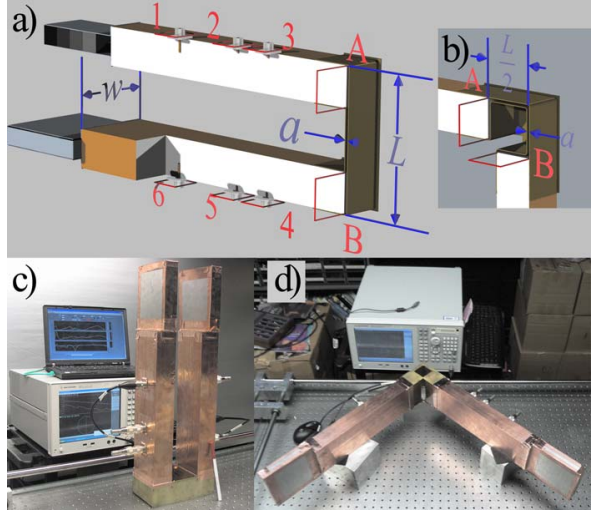

FIG. 1. (Color online) Cut-away schematic showing the experimental geometry for the $180^{\circ}$ bend [panel (a)] with $90^{\circ}$ bend in inset [panel (b)], consisting of an ultranarrow channel connecting two much thicker Teflon waveguide sections and the system of probes employed to perform the measurement. Photos of one particular geometry for the $180^{\circ}$ bend and the $90^{\circ}$ bend are shown in panels (c) and (d), respectively.

the ENZ metamaterial. ${ }^{9}$ In the following, we present our recent experimental and numerical results related to the addition of sharp and arbitrary bends in the narrow waveguide channel "supercoupling" two much larger waveguide sections. As in our previous work, ${ }^{10}$ here we employ a channel at cutoff to mimic the long wavelength characteristics of ENZ materials. We underline that the advantage of using this technique to realize the required ENZ effect does not only reside in the inherent simplicity of the design, but also in the avoidance of major causes of losses from the effects of disorder and imperfections in the experimental realization of even properly designed resonant inclusions. ${ }^{9}$

\section{EXPERIMENTAL SETUP AND REALIZATION}

Our experimental setup contains three sections as shown in Fig. 1, consisting of an ultranarrow channel sandwiched
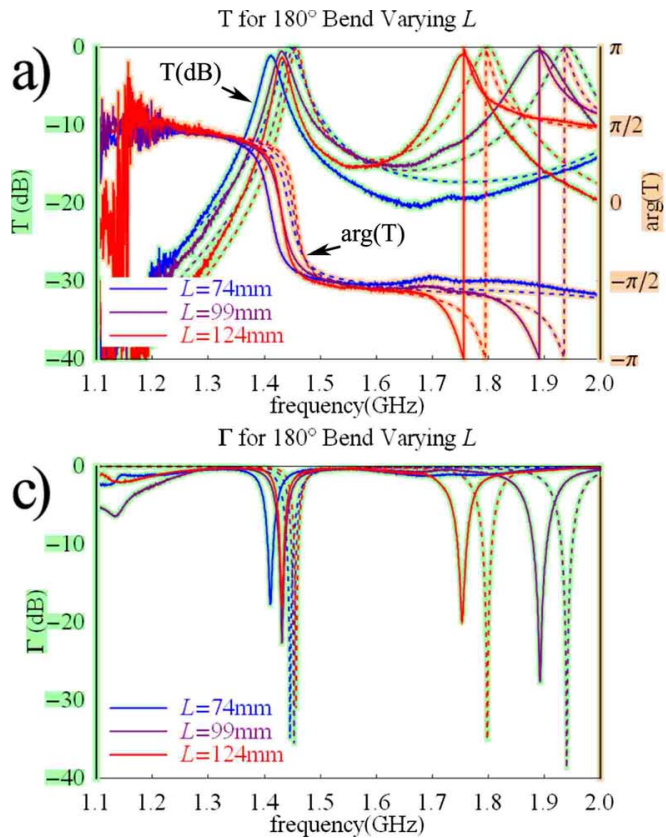

between two copper coated Teflon $\left(\varepsilon=2.0 \varepsilon_{0}\right)$ waveguides. Both of these waveguides have a height of $h=50.8 \mathrm{~mm}$ and an H-plane width of $w=101.4 \mathrm{~mm}$, designed so that the fundamental $\mathrm{TE}_{10}$ mode has its cutoff at frequency of $f$ $=1.04 \mathrm{GHz}$ with monomodal propagation until $2.09 \mathrm{GHz}$. The narrow channel has the same H-plane width, but is filled with air, ensuring a higher cutoff frequency at $f_{0}$ $=1.48 \mathrm{GHz}$. Three probes have been inserted into each waveguide to extract the reflection from and the transmission through the narrow channel connecting them. Within the left waveguide, probe 1 is used to generate the $\mathrm{TE}_{10}$ mode, while the smaller probes 2 and 3 are used to sample the local electric field. The signals collected at 2 and 3 are then used to reconstruct the impinging and reflected modes referenced to plane A. Within the second waveguide, probes 4 and 5 perform the same function, de-embedding the sampled signals into transmitted and reflected modes referenced to plane B. Alternatively and symmetrically, the wave may be excited from the other side of the setup at probe 6 . These eight signals (four obtained when the excitation is at probe 1 and four when it is at probe 6) can then be used to determine the four scattering parameters of the channel. It is worth noting that this extraction procedure neither requires that the feeding probe is perfectly matched to the waveguide, nor that the far ends of the waveguide are well terminated, since at each frequency the six probes may allow us to extract the complex value of the impinging, reflected and transmitted waves through the channel with good accuracy over a wide range of frequencies without de-embedding the individual probes. The detailed extraction procedure is described in the supplemental materials.

All measurements were performed using an Agilent ENA 5071C vector network analyzer. This setup and reconstruction procedure are analogous to our previous experi-

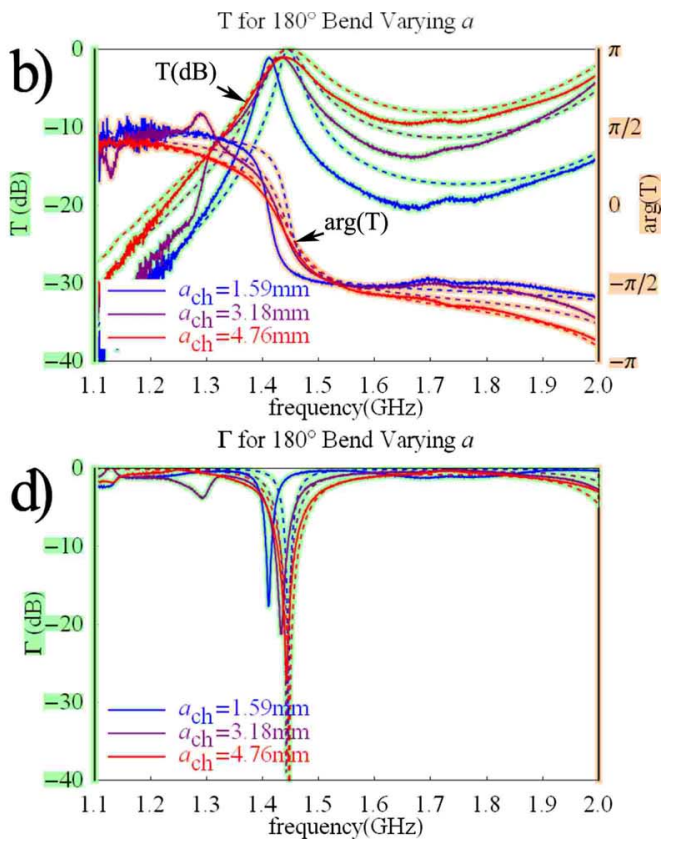

FIG. 2. (Color online) Experimental (solid) and simulation (dashed) results for the $180^{\circ}$ bend. Transmission [panels (a) and (b)] and reflection [panels (c) and (d)] measurements varying the length $L$ of the ENZ channel [panels (a) and (c)] and the height $a$ of the channel [panels (b) and (d)]. Regardless of geometry, there is constantly a peak when the narrow channel is at cut off and behaves effectively as an ENZ material. The phase variation across the channel [panels (a) and (b)] at this frequency is always very small, confirming the ENZ operation of the channel. 

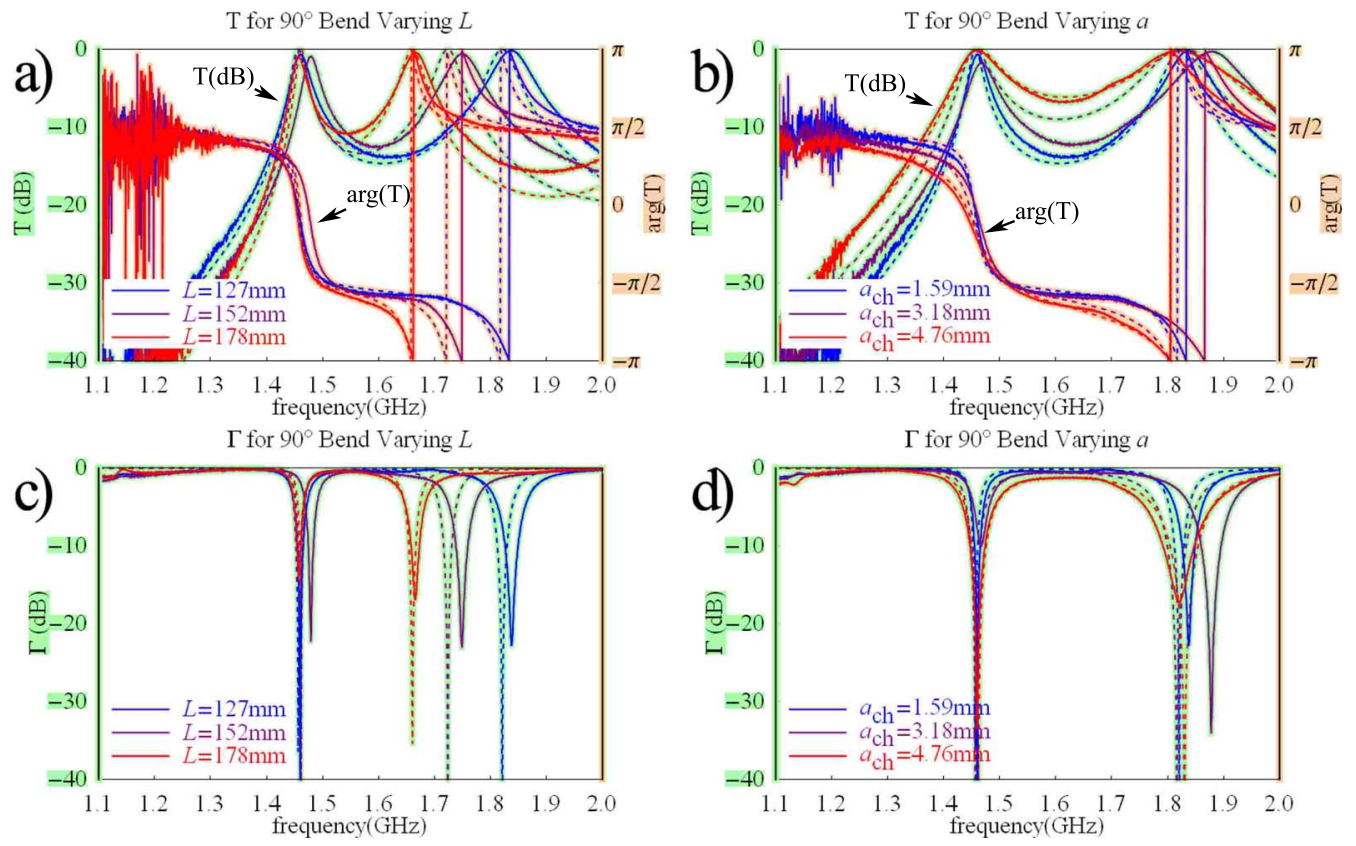

FIG. 3. (Color online) Similar to Fig. 2, but for the $90^{\circ}$ bend configuration.

mental setup, ${ }^{10}$ where the narrow channel was straight and coplanar with the connected waveguide sections. Here, however, we concentrate on the possibility of arbitrarily bending the channel, as previously theoretically suggested. ${ }^{8,9}$ Moreover, we have also improved the realization of the experimental setup, eliminating some imperfections in the connection between the different parts, which caused non-negligible losses.

\section{RESULTS AND DISCUSSION}

A total of ten different channels have been designed, constructed and tested, five examining $90^{\circ}$ bends and five examining $180^{\circ}$ bends. For both geometries, we have analyzed the effects of varying the length $L$ and the height $a$ of the channel. Additionally, full-wave simulations were performed to validate the experimental measurements. ${ }^{15}$

The measured and simulated transmission and reflection coefficients are reported in Fig. 2 for the $180^{\circ}$ bend and in Fig. 3 for the $90^{\circ}$ bend. It is noticed that indeed, regardless of

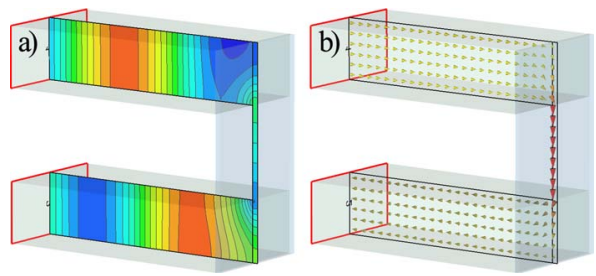

FIG. 4. (Color online) Simulations showing the fields internal to the $180^{\circ}$ bend. Snapshot in time of the normal real part of the magnetic field [panel (a)] and real part of Poynting vector [panel (b)]. In panel (a) warmer colors correspond to positive values while cooler colors correspond to negative values. The channel width has been intentionally left relatively large for clarity, leading to a nonzero phase difference across the ENZ region. However, the fast-wave properties of the channel are very evident also in this configuration. Narrower channels (as those experimentally realized and corresponding to Figs. 2 and 3) would provide an even smaller phase delay and faster wave propagation across the channel at the supercoupling frequency. the geometry $\left(90^{\circ}\right.$ bend or $180^{\circ}$ bend), the length $L$, and the height $a$, there is consistently an amplitude peak in the transmission coefficient and a substantial drop in the reflection at the cutoff frequency $f_{0}$ of the channel in its ENZ operation. Remarkably different from the Fabry-Perot transmission resonances seen at higher frequencies, which are predictably shifted by a change in the channel length, and possibly also by its geometry, the supercoupling resonant frequency is not sensibly shifted by such variations. Changes in the height $a$ affect only the bandwidth of the transmission peaks, with smaller heights yielding narrower peaks, due to the higher resonance $Q$ factor. These results are consistent with the straight channel, ${ }^{10}$ showing how the ENZ supercoupling effect and energy squeezing are not affected by bending or shape variations in the connecting channel, since it relies on a staticlike resonance for which the effective wavelength in the channel is extremely large. Also the phase distribution confirms the ENZ response of the narrow waveguide channel at cutoff: regardless of the geometry, length $L$, or height $a$, the phase difference between the entrance and exit planes is always near zero.

Further evidence on the near zero phase variation through the ENZ region is given through the simulation results of this geometry, reported in Fig. 4 for the $180^{\circ}$ bend and Fig. 5 for the $90^{\circ}$ bend, wherein we see none of the field peaks and nulls associated with regular Fabry-Perot resonant

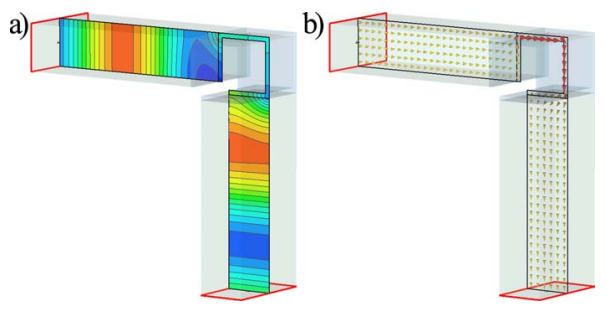

FIG. 5. (Color online) Similar to Fig. 4, but for the $90^{\circ}$ bend 


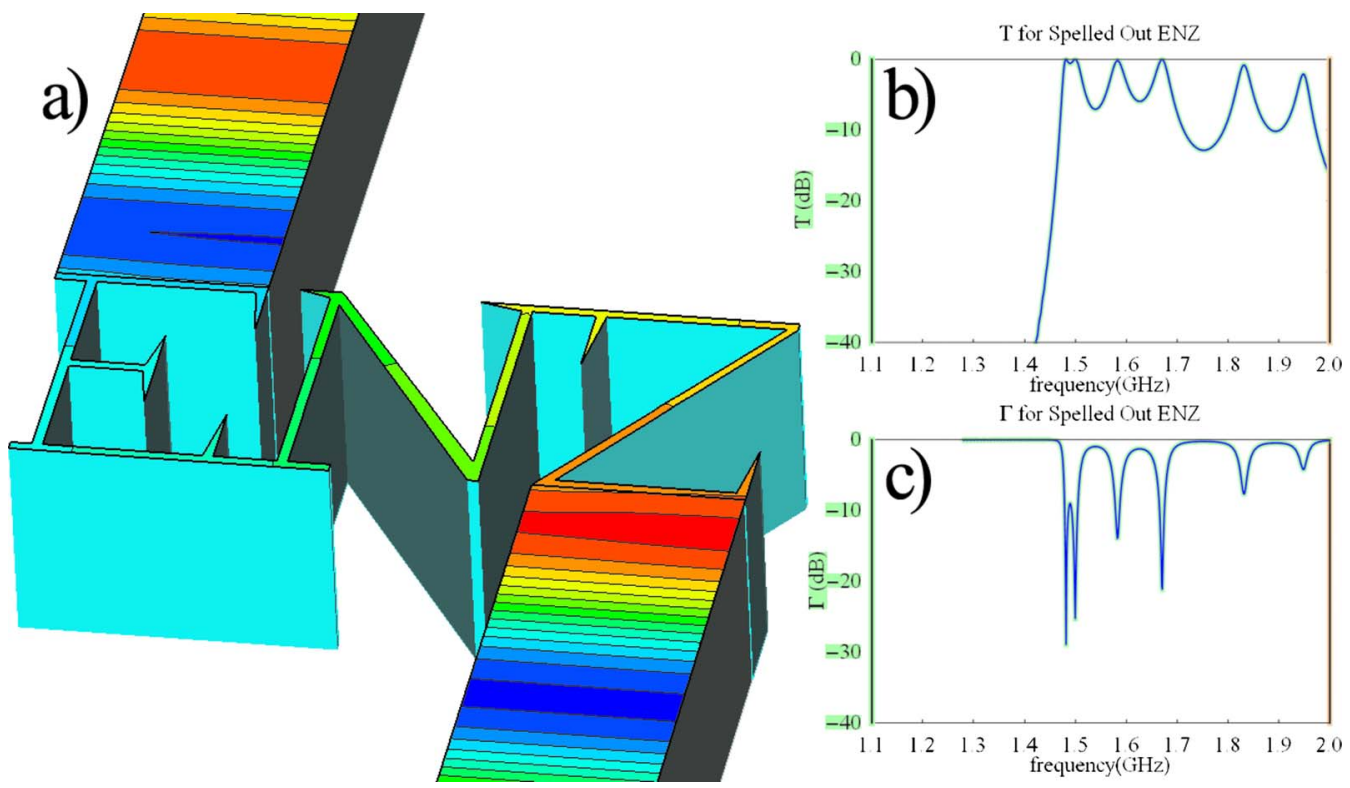

FIG. 6. (Color online) Simulation showing the real part of the magnetic field [panel (a), snapshot in time] in an ultranarrow waveguide channel spelling out the letters ENZ and connecting two much thicker waveguide sections. Warmer colors correspond to positive values while cooler colors correspond to negative values. The plot confirms unity transmission and ultrafast wave propagation along the channel at the ENZ frequency, despite many turns, stubs, $\sim 2.5$ free-space wavelengths of length at the cutoff frequency of the channel. The energy is squeezed into a channel at $\sim 1 / 20$ th of the original waveguide thickness. Transmission [panel (b)] and reflection [panel (c)] coefficients for the same geometry. The frequency relative to panel (a) corresponds to the first transmission peak.

transmission. A plot of the Poynting vector is also reported, demonstrating how the energy squeezes into a region with a height of $3 / 32$ nd of the Teflon waveguide. This channel height was used for its clarity in the figure. Experiments were performed with even lower values to yield a ratio between the heights of 1:32. Consistently with what expected theoretically, 6,7 these narrower channels indeed yield even smaller phase variation.

As a final example of the robustness and flexibility of the ENZ operation, we report in Fig. 6 a numerical simulation for a more dramatic example of a bent channel, wherein the letters "ENZ" are spelled out along the ultranarrow channel. Due to its length and irregularities, there are many FabryPerot peaks between 1.1 and $2.0 \mathrm{GHz}$. However, the field profile shown in the figure, corresponding to the same ENZ frequency $f_{0}$ as in the previous examples, demonstrates unity transmission and near zero phase difference between the entrance and exit faces, despite the complex bending, channel profile and its large cross-section mismatch compared to the outer waveguide sections. The presented results support the versatility of the ENZ concepts and their possible application in complicated routing and supercoupling effects.

\section{CONCLUSIONS}

In this work we have experimentally demonstrated that a narrow ENZ channel, as described in Refs. 6 and 7, can indeed provide supercoupling abilities in spite of sharp bends both at the midplane of the channel and at the entrance and exit faces. The ENZ nature of the channel has been realized by taking advantage of the natural dispersive properties of a rectangular waveguide. This has provided unity transmission at the cutoff frequency of the channel despite sharp bends and both variations in the channel length and width. We believe that the present experimental findings may have interesting implications for energy rerouting and supercoupling through arbitrarily bent ultranarrow channels and for sensing devices. ${ }^{16}$ Moreover, extension and future experimental verification of these concepts at the IR and optical frequencies are within the realms of available technology, paving the way to a wide range of intriguing possibilities for enhanced light transmission through arbitrarily bent plasmonic channels. ${ }^{17}$

${ }^{1}$ A. Alù and N. Engheta, Phys. Rev. E 72, 016623 (2005).

${ }^{2}$ S. Enoch, G. Tayeb, P. Sabouroux, N. Guerin, and P. Vincent, Phys. Rev. Lett. 89, 213902 (2002).

${ }^{3}$ A. E. Serebryannikov, T. Magath, K. Schuenemann, and O. Y. Vasylchenko, Phys. Rev. B 73, 115111 (2006).

${ }^{4}$ R. W. Ziolkowski, Phys. Rev. E 70, 046608 (2004).

${ }^{5}$ A. Alù, M. G. Silveirinha, A. Salandrino, and N. Engheta, Phys. Rev. B 75, 155410 (2007)

${ }^{6}$ M. Silveirinha and N. Engheta, Phys. Rev. Lett. 97, 157403 (2006).

${ }^{7}$ M. G. Silveirinha and N. Engheta, Phys. Rev. B 76, 245109 (2007).

${ }^{8}$ A. Alù, M. G. Silveirinha, and N. Engheta, Phys. Rev. E 78, 016604 (2008).

${ }^{9}$ Q. Cheng, R. Liu, D. Huang, T. J. Cui, and D. R. Smith, Appl. Phys. Lett. 91, 234105 (2007).

${ }^{10}$ B. Edwards, A. Alù, M. E. Young, M. Silveirinha, and N. Engheta, Phys. Rev. Lett. 100, 033903 (2008).

${ }^{11}$ W. Rotman, IEEE Trans. Antennas Propag. 10, 82 (1962).

${ }^{12}$ R. Marqués, J. Martel, F. Mesa, and F. Medina, Phys. Rev. Lett. 89, 183901 (2002).

${ }^{13}$ J. D. Baena, L. Jelinek, R. Marqués, and F. Medina, Phys. Rev. B 72, 075116 (2005).

${ }^{14}$ S. Hrabar, J. Bartolic, and Z. Sipus, IEEE Trans. Antennas Propag. 53, 110 (2005).

${ }^{15}$ CST Studio Suite 2006b. www.cst.com.

${ }^{16}$ A. Alù and N. Engheta, Phys. Rev. B 78, 045102 (2008).

${ }^{17}$ A. Alù and N. Engheta, Phys. Rev. B 78, 035440 (2008) 